\title{
Estudo de um sistema de eletrificação de gotas em pulverizador costal pneumático pelo método de gaiola de Faraday ${ }^{1}$
}

\author{
Rafael Marcão Tavares ${ }^{2}$, João Paulo Arantes Rodrigues da Cunha ${ }^{2}$, Thales Cassemiro Alves ${ }^{2}$, \\ Guilherme Sousa Alves ${ }^{2}$, João Eduardo Ribeiro Silva ${ }^{2}$
}

10.1590/0034-737X201764050004

\begin{abstract}
RESUMO
A aplicação inadequada de agrotóxicos pode causar sérios problemas ao ambiente e o emprego de gotas com pequeno diâmetro pode favorecer a cobertura dos alvos, porém desde que ela aconteça em condições especiais para minimizar a deriva. Objetivou-se, com este trabalho, analisar os fatores que influenciam a relação carga/massa $(\mathrm{Q} / \mathrm{M})$ gerada por um sistema de eletrificação de gotas em um pulverizador pneumático costal com kit eletrostático por meio de uma gaiola de Faraday. Montaram-se dois experimentos, o primeiro em delineamento inteiramente casualizado, a fim de verificar a influência da distância entre o pulverizador e o alvo, a vazão de aplicação e a calda de pulverização, no funcionamento do sistema de eletrificação de gotas. Neste experimento, avaliaram-se quatro distâncias $(0 ; 0,5 ; 1,0$ e 2,0 $\mathrm{m}$ ) entre o pulverizador e a gaiola. No segundo experimento, em esquema fatorial, analisaram-se cinco composições de caldas e cinco vazões $\left(0,25 ; 0,76 ; 1,00 ; 1,45 ;\right.$ e $\left.1,58 \mathrm{~L} \mathrm{~min}^{-1}\right)$. A relação $\mathrm{Q} / \mathrm{M}$ foi determinada por meio da medição da corrente elétrica a partir do volume de calda pulverizado em gaiola de Faraday com um multímetro, dividida pela respectiva massa de líquido. Analisou-se, também, o espectro de gotas produzidas pelo pulverizador, em analisador de partículas por difração de raio laser. Verificou-se que, com o aumento da vazão de pulverização na gaiola de Faraday, menor foi a relação $\mathrm{Q} / \mathrm{M}$, o que também ocorreu com a distância do bocal do pulverizador eletrostático da entrada da gaiola. Em relação à calda de pulverização, a relação $\mathrm{Q} / \mathrm{M}$ aumentou com a adição de óleo mineral, do óleo vegetal e de inseticida. Concluiu-se que a distância entre o bocal do pulverizador e o alvo, a vazão e a calda de aplicação influenciam na eficiência da pulverização eletrostática.
\end{abstract}

Palavras-chave: pulverização eletrostática; tecnologia de aplicação; relação Q/M.

\section{ABSTRACT}

\section{Study of a droplet electrification system in a backpack sprayer in a Faraday cage}

This study evaluated the factors affecting the charge/mass ratio $(\mathrm{Q} / \mathrm{M})$ generated by a droplet electrification system in a backpack sprayer with an electrostatic kit, using a Faraday cage. Two experiments were conducted in completely randomized design to determine the effect of application rate, the distance between the nozzle and the target, and spraying mixtures on performance of the droplet electrification system. The first study evaluated four distances $(0,0.5,1.0$, and $2.0 \mathrm{~m})$ between the nozzle and the Faraday cage; the second study was conducted in a factorial design, with five tank mixtures and five flow rates $\left(0.25,0.76,1.00,1.45\right.$, and $\left.1.58 \mathrm{~L} \mathrm{~min}^{-1}\right)$. The $\mathrm{Q} / \mathrm{M}$ ratio was determined using a multimeter by measuring the electric current from the volume of mixture sprayed in the Faraday cage, which was divided by the respective liquid mass. Droplet spectrum was analyzed by a particle analyzer using laser diffraction. The $\mathrm{Q} / \mathrm{M}$ ratio became smaller as the spraying flow rate increased, similarly as observed with increasing distances of the electrostatic nozzle from the cage entrance. The Q/M ratio increased with the addition of mineral oil, vegetable oil, and insecticide to the spraying mixture. Flow rate, distance between the nozzle and the target, and the spraying mixture affected the electrostatic spraying efficiency.

Key words: electrostatic spraying; application technology; Q/M ratio.

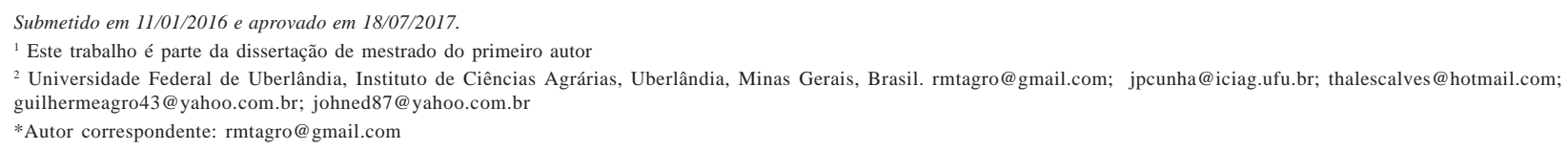

Rev. Ceres, Viçosa, v. 64, n.5, p. 476-485, set/out, 2017 


\section{INTRODUÇÃO}

A aplicação inadequada de agrotóxicos pode causar sérios problemas ao ambiente, deixar resíduos nos alimentos e aumentar o risco de intoxicação dos trabalhadores rurais. Para o sucesso nessas aplicações, uma alternativa é o emprego de gotas com diâmetros reduzidos, a fim de favorecer a cobertura dos alvos. Por apresentarem pequenas massas, essas gotas têm baixa energia cinética, o que facilita a deriva nas pulverizações. Por isso, o emprego dessas gotas deve acontecer em condições especiais, como a pulverização eletrostática.

Essa tecnologia consiste na formação de um campo elétrico que evita o desvio da gota de sua trajetória até o alvo e provoca atração entre ambos, por meio de fornecimento ou extração de elétrons. Desta forma, cargas de mesmo sinal se repelem e cargas de sinais opostos se atraem, além de que a carga de um corpo eletrificado induz uma carga igual e oposta em algum outro corpo condutor aterrado. Então, a nuvem de gotas eletrificadas ao se aproximar da planta, um objeto neutro e aterrado, provoca desequilíbrio entre prótons e elétrons, induz uma carga de sinal contrário na superfície do alvo e, promove assim, atração entre as cargas (Chaim, 2006).

Um dos processos de eletrificação é o de carga por indução com eletrificação indireta, no qual o líquido é mantido aterrado e as gotas adquirem a carga na presença de um intenso campo eletrostático, formado entre o eletrodo de indução, mantido em alta voltagem, e o jato de gotas. O eletrodo de indução deve ser posicionado na região da borda do jato, onde se formam as gotas, a uma distância mínima suficiente, para evitar centelhas de descarga entre o eletrodo e o líquido (Patel et al., 2012).

$\mathrm{O}$ fator que representa a eficiência de um pulverizador eletrostático é a relação carga/massa (Q/M), ou seja, a quantidade de carga elétrica contida em uma massa conhecida de líquido. Essa relação é constatada mediante a divisão da corrente contida no jato de gotas, pela massa de líquido pulverizado e, quanto maior a relação $\mathrm{Q} / \mathrm{M}$, maior a força de atração entre a gota e o alvo (Magno Júnior et al., 2014). Por isso, é importante conhecer os parâmetros que afetam a relação $\mathrm{Q} / \mathrm{M}$ na pulverização eletrostática (Sasaki et al., 2015).

Por essa razão, este trabalho foi conduzido com o objetivo de analisar um sistema de eletrificação de gotas em um pulverizador pneumático costal, a fim de avaliar a influência da distância entre o bocal do pulverizador e o alvo, da composição da calda e da vazão na relação carga/ massa gerada.

\section{MATERIAL E MÉTODOS}

Este trabalho foi realizado no Laboratório de Mecanização Agrícola (LAMEC) da Universidade Federal de
Uberlândia (UFU), Uberlândia, MG. A análise dos espectros de gotas gerados pelo pulverizador utilizado nos experimentos foi realizada no Laboratório de Análise de Tamanho de Partículas (LAPAR), da Faculdade de Ciências Agrárias e Veterinárias, na Universidade Estadual Paulista (UNESP), em Jaboticabal, SP.

Montaram-se dois experimentos, em delineamento inteiramente casualizado, a fim de se verificar a influência da distância entre o pulverizador e o alvo (experimento 1), da vazão de aplicação e da calda de pulverização (experimento 2), no funcionamento de um sistema de eletrificação de gotas, por meio da análise da relação carga/massa (Q/M).

As condições ambientais foram monitoradas durante a realização dos experimentos por meio de um termo-higroanemômetro digital, marca Kestrel ${ }^{\circledR}$ e modelo 4000, com temperaturas entre $21,5^{\circ} \mathrm{C}$ e $29,2^{\circ} \mathrm{C}$, umidade relativa do ar entre 47,9 e $74,0 \%$ e ventos com velocidades entre $1,3 \mathrm{e}$ $4,6 \mathrm{~km} \mathrm{~h}^{-1}$.

O experimento sobre a distância entre o pulverizador e o alvo foi constituído por quatro tratamentos, isto é, as distâncias de $0 ; 0,5 ; 1$ e 2 m, com cinco repetições, com total de 20 parcelas. Nessas pulverizações, utilizou-se apenas água potável e uma vazão média de $0,82 \mathrm{~L} \mathrm{~min}^{-1}$.

O experimento da calda de pulverização foi constituído por um esquema fatorial 5 x 5 , isto é, cinco composições de caldas de pulverização e cinco vazões de aplicação, com quatro repetições e total de 100 parcelas. As caldas avaliadas foram: $\mathbf{A G}$ = água potável; $\mathbf{O M}=$ água potável + óleo mineral (Nimbus, com $42,80 \%$ de i.a., na concentração de $500 \mathrm{~mL}$ para 100 litros de calda); $\mathbf{O V}=$ água potável + óleo vegetal (Veget' oil, com 93,00\% de i.a, na concentração de $500 \mathrm{~mL}$ para 100 litros de calda); $\mathbf{E A}=$ água potável + espalhante adesivo (In-Tec, com 12,0\% do i.a. Nonil Fenol Etoxilado, na concentração de $50 \mathrm{~mL}$ para 100 litros de calda); e IN = água potável + inseticida (Provado 200 SC, com $20 \%$ do i.a. imidacloprido, na concentração de $25 \mathrm{~mL}$ para 100 litros de calda). As vazões avaliadas foram: 0,25; 0,76; 1,00; 1,45 e 1,58 $\mathrm{L} \mathrm{min}^{-1}$.

Previamente às aplicações, avaliaram-se potencial hidrogeniônico $(\mathrm{pH})$, condutividade elétrica, densidade, viscosidade e tensão superficial das cinco caldas utilizadas. Todas as avaliações foram realizadas a partir de soluções preparadas em béqueres de $0,5 \mathrm{~L}$, à temperatura de $25^{\circ} \mathrm{C}$. Foi utilizado delineamento inteiramente casualizado, com quatro repetições para cada uma das caldas analisadas.

$\mathrm{O} \mathrm{pH}$ e a condutividade elétrica foram medidos diretamente nas soluções com peagâmetro e condutivímetro portátil, marca Hach® e modelo HQ40d, previamente calibrado por meio de soluções-padrão. A densidade foi estimada por meio da determinação da massa de $0,1 \mathrm{~L}$ da solução depositada em um balão volumétrico, em balança com 
resolução de $0,1 \mathrm{mg}$. A viscosidade dinâmica foi determinada com um viscosímetro rotativo microprocessado, marca Quimis® e modelo Q860M21, com o rotor zero e rotação de 60 rpm. A tensão superficial foi determinada, após calibração com água destilada, por um tensiômetro de bancada com anel de platina, marca Kruss ${ }^{\circledR}$ e modelo K6.

Para as aplicações, utilizou-se um pulverizador pneumático costal motorizado da marca Stihl@, modelo SR450, com um volume de tanque de 14 litros e um motor dois tempos monocilíndrico de $2900 \mathrm{~W}$ de potência. O fluxo máximo de ar gerado pelo pulverizador é de $920 \mathrm{~m}^{3} \mathrm{~h}^{-1}$, na rotação máxima de 6800 rpm, já que há três acelerações possíveis. Adotou-se em todos os experimentos uma rotação do motor mantida com a aceleração intermediária. Verificou-se, com um multímetro, por meio de uma ponta de prova de alta tensão, que a vela de ignição do motor é alimentada por uma tensão de $7 \mathrm{kV}$. Por se tratar de um motor dois tempos, a corrente gerada a partir da tensão na vela de ignição, apesar de se caracterizar como contínua, apresenta picos de leitura. A corrente passa por uma rede de resistores e diodos para retificação.

Para a pulverização eletrostática, foi instalado ao pulverizador um kit de conversão da marca Spectrum ${ }^{\circledR}$, modelo 3010, com processo de carga por indução com eletrificação indireta, com o que o líquido é mantido aterrado, com voltagem igual a zero.

O bocal do Spectrum 3010 converte o sistema pneumático convencional para um sistema eletrostático, sem necessidade de uma bateria externa como fonte de alimentação elétrica. $\mathrm{O}$ bocal se encaixou sobre a extremidade da saída de ar do pulverizador. No seu interior, na parte superior, há um orifício de plástico não condutor para saída da calda de pulverização, e na parte oposta há um eletrodo condutor. Na parte externa do bocal, para fornecimento da energia eletrostática há um filtro resistorcapacitor e um circuito retificador, ambos ligados por um único fio à vela de ignição do pulverizador. Há também um segundo fio, ligado ao chassi do pulverizador, para estabelecer o aterramento do sistema. Quando se liga o motor do pulverizador, a corrente elétrica é fornecida ao eletrodo condutor e resulta na criação de um campo eletrostático negativo no interior do bocal. Com o orifício de saída da calda e o eletrodo posicionados em paredes opostas do interior do bocal, a corrente de ar e a calda são liberadas simultaneamente, de forma que a corrente de ar eletrifica o líquido e arrasta as gotas para fora do bocal, evitando o escorrimento (Dobbins, 1995). Assim, o kit de conversão eletrostático apresenta o fio ligado à vela de ignição do motor e o bocal de condução eletrostática, ambos acoplados ao pulverizador costal pneumático.

A quantidade de carga do sistema foi determinada pelo método utilizado por Chaim (1998), que consiste no uso de uma gaiola de Faraday. No experimento 2, a saída do bocal do pulverizador foi mantida a $0,05 \mathrm{~m}$ de distância da abertura da gaiola, construída no LAMEC-UFU, com as dimensões de $0,8 \mathrm{~m}$ de diâmetro e $0,6 \mathrm{~m}$ de comprimento, de modo que todo o jato de pulverização fosse captado pela gaiola ao longo dos tratamentos. Para a construção da gaiola, fez-se uma estrutura cilíndrica de aço galvanizado, envolto com tela metálica, malha 6 (abertura de 3,033 $\mathrm{mm}$ ) e fio de 1,2 $\mathrm{mm}$. A gaiola foi isolada por meio de uma haste de madeira de $1,7 \mathrm{~m}$ de comprimento, com $0,7 \mathrm{~m}$ da haste abaixo do nível do solo.

Antes de todas as pulverizações, marcou-se o tempo de $15 \mathrm{~s}$ até se estabilizar o pulverizador e, em seguida, pulverizou-se durante 2 min no interior da gaiola. Adotou-se o volume fixo de $4 \mathrm{~L}$ de calda no tanque do pulverizador. Posteriormente, mensurou-se a quantidade de líquido pulverizada, com uma proveta graduada, com precisão de $5 \mathrm{~mL}$. Determinou-se a densidade de cada calda utilizada, em todos os experimentos, por meio da relação entre a massa da calda e um volume amostrado. Dessa forma, a cada pulverização no interior da gaiola, determinou-se a massa de líquido pulverizada.

Para verificar a corrente elétrica presente nas gotas pulverizadas, conectou-se à gaiola um multímetro da marca Minipa ${ }^{\circledR}$, modelo ET-2517A, com escala de 0 a $600 \mu \mathrm{A}$ e precisão de $\pm 0,2 \%$. O multímetro foi aterrado por uma barra de ferro enterrada a $1 \mathrm{~m}$ abaixo do nível do solo (Maski \& Durairaj, 2010). As leituras no multímetro foram feitas no modo de corrente elétrica contínua. Dessa forma, determinou-se a descarga de eletricidade contida no jato de gotas, a fim de verificar a relação carga/massa $(\mathrm{Q} /$ $\mathrm{M})$, em $\mathrm{mC} \mathrm{kg}^{-1}$, por meio da relação entre a corrente elétrica $\left(\mathrm{mC} \mathrm{s}^{-1}\right)$ e a quantidade da massa de líquido pulverizada $\left(\mathrm{kg} \mathrm{s}^{-1}\right)$.

Também foi realizada a análise do espectro de gotas pulverizadas, com o sistema eletrostático ligado, por meio de um analisador de partículas em tempo real Mastersizer S® (Malvern Instruments Ltd.). Essa análise foi feita em delineamento inteiramente casualizado, repetindo-se o fatorial 5 x 5 do experimento 2 .

Inicialmente, verificou-se o alinhamento do feixe óptico para garantir o seu correto posicionamento no sistema detector, corrigiram-se possíveis contaminações das lentes por partículas e procedeu-se à calibração do aparelho. Para medição do espectro de gotas, utilizou-se uma lente focal de $300 \mathrm{~mm}$, capaz de analisar gotas na faixa de 0,5 a 900 ìm. $O$ bocal do pulverizador foi mantido na horizontal a $35 \mathrm{~cm}$ de distância do feixe de laser.

As condições ambientais durante a realização dos experimentos foram: temperatura entre $26,2^{\circ} \mathrm{C}$ e $27,1^{\circ} \mathrm{C}$, umidade relativa do ar entre 67 e $83 \%$ e ausência de ventos e de luminosidade externa.

Avaliaram-se o diâmetro da mediana volumétrica (DMV), a amplitude relativa (AR) e a percentagem do vo- 
lume de gotas pulverizadas com diâmetro inferior a 100 $\mu \mathrm{m}(\mathrm{Dv}<100 \mathrm{ìm})$.

Todos os dados foram primeiramente submetidos aos testes de normalidade de Shapiro-Wilk e de KolmogorovSmirnov, e de homogeneidade das variâncias de Levene, ambos a 0,01 de significância, com o programa SPSS 20. Nos casos em que as pressuposições não foram atendidas, os dados foram transformados em $\sqrt{\mathbf{x}}$ e submetidos à nova análise. Somente quando a transformação corrigiu ou pelo menos melhorou uma das pressuposições, sem prejudicar as demais, utilizaram-se os dados transformados para a análise de variância. Do contrário, utilizaramse os dados originais (Sokal \& Rohlf, 1995).

Em todos os experimentos, após a análise das pressuposições, os dados foram submetidos à análise de variância pelo programa estatístico SISVAR 5.3 (Ferreira, 2008). Quando pertinente, os tratamentos qualitativos foram comparados entre si pelo teste de Tukey, a 0,05 de significância, enquanto os tratamentos quantitativos foram submetidos à análise de regressão. As curvas foram elaboradas pelo programa SigmaPlot 12.0.

\section{RESULTADOS E DISCUSSÃO}

Na Figura 1A, é apresentada a variação da relação Q/ $\mathrm{M}$, conforme se distanciou o bocal do pulverizador eletrostático da entrada da gaiola de Faraday. Observou-se, à medida que se distanciou o pulverizador da gaiola, a redução da relação Q/M gerada pela calda eletrificada de pulverização. Optou-se por um modelo linear porque a redução da relação $\mathrm{Q} / \mathrm{M}$ foi constante e observou-se que o aumento de $1 \mathrm{~m}$ na distância entre o pulverizador e a gaiola provocou a redução de 0,0412 vezes da relação $\mathrm{Q} / \mathrm{M}$.

Usando-se a gaiola como referência, percebeu-se que, para alvos mais distantes do bocal de indução eletrostática, a pulverização perde eficiência. Uma possível explicação é que esses resultados podem ser devidos à perda da carga eletrostática durante a trajetória entre o bocal e o alvo. Outra possibilidade pode ser também a perda de calda para o ambiente, por meio de deriva ou evaporação, pois se trata de gotas com diâmetros reduzidos.

A relação $\mathrm{Q} / \mathrm{M}$ e a amplitude relativa (AR) do espectro de gotas, analisadas no experimento 2, são apresentadas na Tabela 1, para as quais não houve interação entre os fatores. Tanto as caldas de pulverização quanto as vazões influenciaram esses parâmetros, que apresentaram diferenças entre si. Este fato mostra que, apesar de a pulverização eletrostática ser influenciada, tanto pela composição das caldas de pulverização, quanto pelas vazões pulverizadas, estes fatores atuam independentemente no sistema eletrostático.

Quanto à calda de pulverização, a relação $\mathrm{Q} / \mathrm{M}$ aumentou com a adição do óleo mineral (OM), do óleo vege- tal (OV) e do inseticida (IN). No entanto, não houve alteração da relação com a adição do espalhante adesivo (EA). Por isso, os componentes da calda de pulverização são fatores relevantes na eficiência da pulverização eletrostática. Entretanto, a alteração da relação Q/M depende da substância adicionada. A adição de adjuvantes à calda reduziu a amplitude relativa em comparação com as pulverizações apenas com água (AG), com exceção da calda IN que não se diferenciou da calda AG.

Ao se alterarem as propriedades da calda, podem também ocorrer alterações dos parâmetros técnicos da pulverização, como constatado por Sasaki et al. (2015). Esses autores, em estudo para avaliar o efeito da adição de adjuvantes à calda de pulverização eletrostática, concluíram que o uso de adjuvantes na pulverização eletrostática pode melhorar a eficiência da tecnologia, pois aumenta a carga elétrica da gota e a força de atração entre o alvo e a gota. Observaram ainda que caldas com produtos que aumentam a condutividade elétrica tendem a apresentar maior relação $\mathrm{Q} / \mathrm{M}$, enquanto produtos que reduzem esta característica causam a diminuição da relação $\mathrm{Q} / \mathrm{M}$, o que não pode ser visto nos resultados encontrados neste trabalho.

Na Figura 1B, é apresentada a variação da relação Q/ $\mathrm{M}$ no experimento 2, na qual se nota que, conforme aumentou-se a vazão de pulverização na gaiola de Faraday, menor foi a eficiência da aplicação. Nesta curva, optou-se por modelo exponencial, pois a redução da relação Q/M é acentuada até estabilizar-se, sem expectativa de crescimento, como visto para as maiores vazões. Este resultado indicou que quanto maior a quantidade de água pulverizada, menor a eficiência do sistema eletrostático, isto é, maior será a carga necessária para eletrificar a calda. $\mathrm{O}$ aumento da vazão levou ao aumento de massa, que diminuiu a relação $\mathrm{Q} / \mathrm{M}$, mostrando que este sistema não proporcionou compensação da energia gerada em função da massa da calda. Estes resultados também foram observados por Magno Júnior et al. (2014), em trabalho para desenvolver um dispositivo eletrostático para pulverizador pneumático costal.

Se, por um lado, as vazões menores resultaram em melhores relações Q/M, por outro, vazões muito baixas podem ser inviáveis em condições de campo, conforme afirmaram Magno Júnior et al. (2014), principalmente em relação ao caminhamento na área. Dependendo da taxa de aplicação almejada, isto é, quanto menor a vazão, mais devagar deve ser o caminhamento do pulverizador a fim de aplicar a mesma taxa. Uma solução para isso seria a redução de taxas de aplicação com a pulverização eletrostática, a fim de proporcionar maior deposição das gotas, alternativa que ainda precisa ser mais bem estudada.

Neste trabalho, encontraram-se as maiores relações Q/M médias por volta de $0,21 \mathrm{mC} \mathrm{kg}^{-1}$, valor que está 
abaixo do nível mínimo de $0,80 \mathrm{mC} \mathrm{kg}^{-1}$, o que torna a pulverização eletrostática mais eficiente, de acordo com Carlton et al. (1995). Isso pode ser explicado pela característica peculiar do sistema eletrostático analisado, que não possui um sistema próprio de geração de tensão, já que a corrente contínua, gerada a partir do motor 2 tempos, apresentava picos. As leituras do multímetro foram
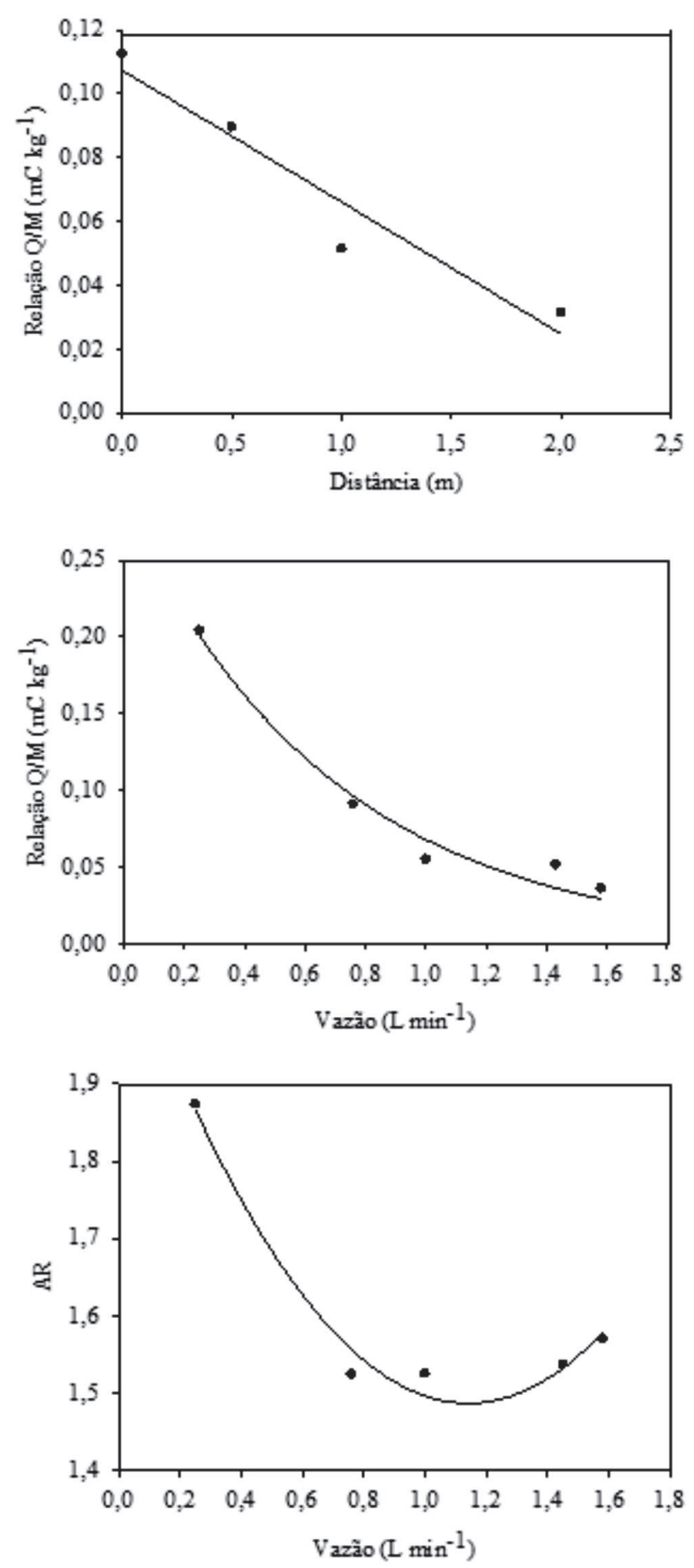

B. Relação Q/M em função da vazão do pulverizador. $\mathrm{Fc}=$ $197,15^{*}$; C.V. $(\%)=24,79 ; \mathrm{W} / \mathrm{K}-\mathrm{S}=\mathbf{0 , 9 8 2} ; \mathrm{F}_{\text {Levene }}=3,616$.

C. Amplitude relativa do espectro de gotas em função da vazão. $\mathrm{Fc}=181,17 * ; \mathrm{C} . \mathrm{V} .(\%)=3,11 ; \mathrm{W} / \mathrm{K}-\mathrm{S}=0,631 ; \mathrm{F}_{\text {Levene }}$ $=5,966$.

valor de F calculado. C.V: coeficiente de variação. W/K-S; $F_{\text {Levene }}$ : estatística dos testes de Shapiro-Wilk ou de Kolmogorov-Smirnov, e de Levene, respectivamente. Valores em negrito indicam resíduos com distribuição normal e variâncias homogêneas, a 0,01 de significância. * significativo a 0,05 .

Figura 1: Relação Q/M em função da distância entre o alvo e a ponta de pulverização (A) e da vazão (B); e amplitude relativa (AR) em função da vazão (C).

Rev. Ceres, Viçosa, v. 64, n.5, p. 476-485, set/out, 2017 
bocal de um pulverizador eletrostático na copa de árvores de maçãs, também demostraram que valores inferiores a $0,8 \mathrm{mC} \mathrm{kg}^{-1}$ podem causar aumento da deposição no alvo. Estes autores trabalharam com uma relação Q/ M de $0,28 \mathrm{mC} \mathrm{kg}^{-1}$, a partir de uma tensão de $4 \mathrm{kV}$ e vazão de $0,56 \mathrm{~L} \mathrm{~min}^{-1}$.

Na Figura 1C, é apresentada a amplitude relativa (AR) do espectro de gotas gerado pelas pulverizações no experimento 2, a qual foi maior ao se pulverizar com a menor vazão. Sobre isso, Sasaki et al. (2015) afirmaram que o espectro deve ser o mais homogêneo possível, de forma que quanto mais próximo de zero for a $A R$, mais homogêneo é o espectro e maiores as chances de atingir o alvo e reduzir perdas.

$\mathrm{O} \mathrm{pH}$, a condutividade elétrica, a densidade, a viscosidade dinâmica e a tensão superficial de todas as caldas analisadas no experimento 2 estão apresentados na Tabela 2. Em geral, a adição de produtos às caldas aumentou todos os parâmetros, quando se compara com os da calda AG. O pH ficou entre 7,64 e 7,99, sendo que apenas a calda OV caracterizou-se com um $\mathrm{pH}$ semelhante ao da calda AG. A condutividade elétrica foi menor que a da calda AG apenas na calda OV e o maior aumento foi observado na calda EA. A densidade e a viscosidade dinâmica da calda AG foi semelhante à das caldas EA e IN, respectivamente. A tensão superficial foi menor nas caldas OM e EA.

As interações entre os fatores caldas e vazões, em relação ao diâmetro da mediana volumétrica (DMV) e à percentagem do volume de gotas pulverizadas com diâmetro inferior a 100 ìm ( Dv < 100 ì m), estão apresentadas na Tabela 3. Para as caldas de aplicação, a classificação das médias ocorre com letras minúsculas, enquanto a comparação das vazões ocorre por meio das curvas de regressão.

Tabela 1: Relação Q/M gerada pela pulverização eletrostática e amplitude relativa (AR) do espectro de gotas de diferentes das caldas

\begin{tabular}{lcc}
\hline Caldas & Relação Q/M $\left(\mathbf{m C ~ k g}^{-1}\right)$ & AR \\
\hline AG & $0,072 \mathrm{~b}$ & $1,658 \mathrm{c}$ \\
OM & $0,095 \mathrm{a}$ & $1,561 \mathrm{a}$ \\
OV & $0,096 \mathrm{a}$ & $1,579 \mathrm{a}$ \\
$\mathrm{EA}$ & $0,081 \mathrm{ab}$ & $1,597 \mathrm{ab}$ \\
$\mathrm{IN}$ & $0,094 \mathrm{a}$ & $1,640 \mathrm{bc}$ \\
\hline $\mathrm{Fc}$ & $4,87^{*}$ & $13,52^{*}$ \\
$\mathrm{~W} / \mathrm{K}-\mathrm{S}$ & $\mathbf{0 , 9 8 2}$ & 0,631 \\
$\mathrm{~F}_{\text {Levene }}$ & 3,616 & 5,966 \\
C.V.(\%) & 24,79 & 3,11 \\
\hline
\end{tabular}

Médias seguidas por letras distintas, nas colunas, diferem entre si pelo teste de Tukey a 0,05 . Fc; W/K-S; $F_{\text {Levene }}$ : valor do F calculado; estatística dos testes de Shapiro-Wilk e de Kolmogorov-Smirnov, e de Levene, respectivamente. C.V: coeficiente de variação. Valores em negrito indicam resíduos com distribuição normal a 0,01. *significativo a 0,01 .
A adição de substâncias à água reduziu o diâmetro das gotas pulverizadas, em comparação com o da calda AG. Em relação à percentagem de gotas com diâmetros menores que $100 \mu \mathrm{m}$, a calda AG apresentou menores valores de gotas pulverizadas com diâmetros reduzidos. Nota-se que a adição de substâncias à calda de pulverização, ao reduzir o diâmetro das gotas pulverizadas, aumenta a percentagem de gotas menores que $100 \mu \mathrm{m}$. Quanto a esses parâmetros, a adição de óleo mineral determinou maiores diferenças em comparação com os da calda apenas com água, ao gerar gotas com os menores DMVs.

O efeito da adição de substâncias depende da composição química e da formulação da calda, como foi concluído por Cunha et al. (2010), que, ao avaliarem os efeitos da temperatura da calda e da adição de adjuvantes de uso agrícola, nas características físico-químicas de soluções aquosas para aplicação de agrotóxicos, concluíram que o comportamento dessas características não é semelhante mesmo para produtos com mesma indicação de uso.

Sasaki et al. (2015) observaram que, em geral, a adição de adjuvantes à calda de pulverização eletrostática tem tendência de reduzir o diâmetro das gotas. No entanto, nem todas as substâncias analisadas atuaram dessa forma. Por isso, Cunha et al. (2010) defendem que o efeito da adição de um adjuvante não pode ser generalizado, já que a tecnologia para quebra de gotas também influencia na caracterização do espectro. No trabalho de Sasaki et al. (2015), o sistema de fragmentação de líquido foi o pneumático, assim como o deste trabalho, o que influenciou os resultados, de acordo com os autores. Além disso, para Sasaki et al. (2015), o método de carregamento elétrico e a geometria do eletrodo do sistema eletrostático também podem influenciar no espectro de gotas, o que pode explicar os resultados aqui obtidos.

Na Figura 2, são apresentados o diâmetro da mediana volumétrica (DMV) e a percentagem do volume de gotas pulverizadas com diâmetro inferior a 100 ìm (Dv < 100 ìm, $\%$ ), para cada uma das caldas analisadas. Na Tabela 4, estão apresentados as equações, os coeficientes de determinação $\left(\mathrm{R}^{2}\right)$, os pontos críticos e o valor de $\mathrm{F}$ calculado para esses modelos das regressões.

A partir dessas curvas, em geral, notou-se que os DMVs foram maiores conforme aumentaram as vazões e, consequentemente, menores as percentagens de gotas com diâmetros reduzidos.

Resultados semelhantes quanto aos diâmetros das gotas pulverizadas foram obtidos por Magno Júnior et al. (2014). Os autores observaram que, ao aumentar a vazão do pulverizador pneumático, houve aumento no tamanho de gotas, já que a quantidade de ar gerada pela turbina permaneceu constante para fragmentar uma massa maior de líquido. Além disso, a adição de produtos à calda reduziu o diâmetro das gotas pulverizadas, uma vez que a cal- 
da AG apresentou os maiores DMVs e as menores percentagens de gotas com diâmetros menores que 100 ìm . A calda EA, por sua vez, foi a que apresentou a maior quantidade de valores semelhantes ou próximos aos da calda AG. Por outro lado, a calda OM foi a que gerou gotas com os menores diâmetros.

Estes resultados mostram que as alterações do espectro de gotas também influenciaram na eficiência da pulverização eletrostática, pois, com a redução das vazões e dos diâmetros de gotas pulverizadas, houve aumento da relação Q/M. Dessa forma, há indicação de que, além da composição da calda e da massa de líquido pulverizado, a dimensão das gotas também afetou a pulverização eletrostática, ou seja, gotas com diâmetros menores exigem menores quantidades de carga para sua eletrificação. Há que salientar que, no equipamento analisado, ao se alterar a vazão, também ocorre modificação do espectro de gotas gerado.

Tabela 2: Propriedades físico-químicas da água potável (AG), água potável + óleo mineral (OM), água potável + óleo vegetal (OV), água potável + espalhante adesivo (EA) e água potável + inseticida (IN)

\begin{tabular}{|c|c|c|c|c|c|}
\hline \multirow{2}{*}{ Caldas } & \multirow{2}{*}{ pH } & \multicolumn{3}{|c|}{ Cond. elétricaDensidade Viscosidade dinâmica } & \multirow{2}{*}{$\begin{array}{c}\text { Tensão superficial } \\
\left(\mathrm{mN} \mathrm{m}^{-1}\right)\end{array}$} \\
\hline & & $\left(\mu \mathrm{S} \mathbf{c m}^{-1}\right)$ & $\left(\mathrm{g} \mathrm{cm}^{-3}\right)$ & $(\mathrm{mPa} \mathrm{s})$ & \\
\hline$\overline{A G}$ & $7,81 \mathrm{~b}$ & $18,25 \mathrm{c}$ & $1,00 \mathrm{c}$ & $2,00 \mathrm{~d}$ & $80,90 \mathrm{~d}$ \\
\hline $\mathrm{OM}$ & $7,99 \mathrm{a}$ & $18,42 \mathrm{~b}$ & $0,97 \mathrm{ab}$ & $4,14 \mathrm{~b}$ & $45,12 \mathrm{a}$ \\
\hline $\mathrm{OV}$ & $7,74 \mathrm{~b}$ & $18,05 \mathrm{~d}$ & $0,95 \mathrm{a}$ & $4,43 \mathrm{a}$ & $46,92 \mathrm{~b}$ \\
\hline EA & $7,64 \mathrm{c}$ & $18,98 \mathrm{a}$ & $0,98 \mathrm{bc}$ & $3,13 \mathrm{c}$ & $44,72 \mathrm{a}$ \\
\hline IN & $7,95 \mathrm{a}$ & $18,42 \mathrm{~b}$ & $0,96 \mathrm{ab}$ & $2,10 \mathrm{~d}$ & $60,97 \mathrm{c}$ \\
\hline $\mathrm{Fc}$ & $42,99 *$ & $100,56^{*}$ & $7,76^{*}$ & $746,61 *$ & $2756,55^{*}$ \\
\hline W & 20,239 & 3,636 & 1,368 & 2,342 & 1,984 \\
\hline $\mathrm{F}_{\text {Levene }}$ & 0,946 & 0,892 & 0,950 & 0,903 & 0,931 \\
\hline C.V. $(\%)$ & 0,56 & 0,37 & 1,36 & 2,59 & 1,07 \\
\hline
\end{tabular}

Médias seguidas por letras distintas, nas colunas, diferem entre si pelo teste de Tukey a $0,05 . \mathrm{Fc} ; \mathrm{W} ; \mathrm{F}_{\mathrm{Levene}}$ : valor do F calculado; estatísticas dos testes de Shapiro-Wilk e de Levene, respectivamente. C.V: coeficiente de variação. Valores em negrito indicam resíduos com distribuição normal e variâncias homogêneas a 0,01 . *significativo a 0,01 .

Tabela 3: Diâmetro da mediana volumétrica (DMV) e percentagem do volume de gotas menores do que 100 ìm (Dv < 100 ìm) de diferentes tipos de caldas e vazões na pulverização eletrostática

\begin{tabular}{|c|c|c|c|c|c|c|}
\hline \multicolumn{7}{|c|}{ MV $(\mu \mathrm{m})$} \\
\hline \multirow{2}{*}{ Caldas } & \multicolumn{6}{|c|}{ Vazões $\left(L\right.$ min $\left.^{-1}\right)$} \\
\hline & 0,25 & 0,76 & 1,00 & 1,45 & 1,58 & Média \\
\hline $\mathrm{AG}$ & $82,74 \mathrm{c}$ & $92,39 \mathrm{~d}$ & $91,97 \mathrm{c}$ & $93,53 \mathrm{~cd}$ & $95,78 \mathrm{~d}$ & 91,28 \\
\hline $\mathrm{OM}$ & $68,51 \mathrm{a}$ & $71,20 \mathrm{a}$ & $74,94 \mathrm{a}$ & $77,97 \mathrm{a}$ & $85,32 \mathrm{~b}$ & 75,59 \\
\hline OV & $71,46 \mathrm{a}$ & $78,92 \mathrm{~b}$ & $83,67 \mathrm{~b}$ & $83,29 \mathrm{~b}$ & $76,60 \mathrm{a}$ & 78,79 \\
\hline EA & $79,30 \mathrm{bc}$ & $83,75 \mathrm{c}$ & $94,69 \mathrm{c}$ & $89,92 \mathrm{c}$ & $89,50 \mathrm{c}$ & 87,43 \\
\hline IN & $76,50 \mathrm{~b}$ & $81,86 \mathrm{bc}$ & $81,70 \mathrm{~b}$ & $94,20 \mathrm{~d}$ & $87,58 \mathrm{bc}$ & 84,37 \\
\hline Média & 75,70 & 81,62 & 85,39 & 87,78 & 86,95 & \\
\hline \multicolumn{7}{|c|}{$\mathrm{Fc}^{\text {int: }} 14,06^{*} ; \mathrm{W}: 0,932 ; \mathrm{F}_{\text {Levene }}: 2,659 ;$ C.V. $(\%): 2,30$} \\
\hline \multicolumn{7}{|c|}{$\mathrm{Dv}<100 \mu \mathrm{m}(\%)$} \\
\hline \multirow{2}{*}{ Caldas } & \multicolumn{6}{|c|}{ Vazões $\left(L\right.$ min $\left.^{-1}\right)$} \\
\hline & 0,25 & 0,76 & 1,00 & 1,45 & $\mathbf{1 , 5 8}$ & Média \\
\hline AG & 62,49 a & 55,25 a & 55,57 a & $54,34 \mathrm{ab}$ & $52,72 \mathrm{a}$ & 56,07 \\
\hline $\mathrm{OM}$ & $79,04 \mathrm{~d}$ & $75,24 \mathrm{~d}$ & $71,23 \mathrm{c}$ & $67,78 \mathrm{~d}$ & $61,37 \mathrm{c}$ & 70,93 \\
\hline OV & $74,93 \mathrm{c}$ & $66,60 \mathrm{c}$ & $62,76 \mathrm{~b}$ & $63,02 \mathrm{c}$ & $68,53 \mathrm{~d}$ & 67,17 \\
\hline EA & $66,33 \mathrm{~b}$ & $62,47 \mathrm{~b}$ & $53,80 \mathrm{a}$ & $57,26 \mathrm{~b}$ & $57,52 \mathrm{~b}$ & 59,48 \\
\hline IN & $68,75 \mathrm{~b}$ & $63,51 \mathrm{bc}$ & $63,41 \mathrm{~b}$ & $54,02 \mathrm{a}$ & $58,68 \mathrm{bc}$ & 61,67 \\
\hline Média & 70,31 & 64,61 & 61,35 & 59,28 & 59,76 & \\
\hline
\end{tabular}

Médias seguidas por letras distintas, nas colunas, diferem entre si, pelo teste de Tukey a 0,05 . $\mathrm{Fc}^{\text {int: }}$ valor do $\mathrm{F}$ calculado para a interação entre caldas e vazões. W; $\mathrm{F}_{\text {Levene }}$ : estatística dos testes de Shapiro-Wilk e de Levene, respectivamente. C.V: coeficiente de variação. *significativo a 0,01 .

Rev. Ceres, Viçosa, v. 64, n.5, p. 476-485, set/out, 2017 
Apesar de Maski \& Durairaj (2010) e Sasaki et al. (2015) afirmarem que a adição de adjuvantes pode favorecer a eficiência da pulverização eletrostática por meio do aumento da condutividade elétrica das caldas, isto não foi observado neste trabalho. Apesar de os produtos terem aumentado a condutividade elétrica das cal- das em comparação com a da calda AG (Tabela 2), isto não se refletiu na tendência da relação $\mathrm{Q} / \mathrm{M}$, pois a calda EA com maior condutividade elétrica apresentou a relação $\mathrm{Q} / \mathrm{M}$ semelhante à gerada pela calda $\mathrm{AG}$ (Tabela 1). Contudo, embora estatisticamente as caldas tenham se diferenciado quanto à condutividade elétrica, a diferen-
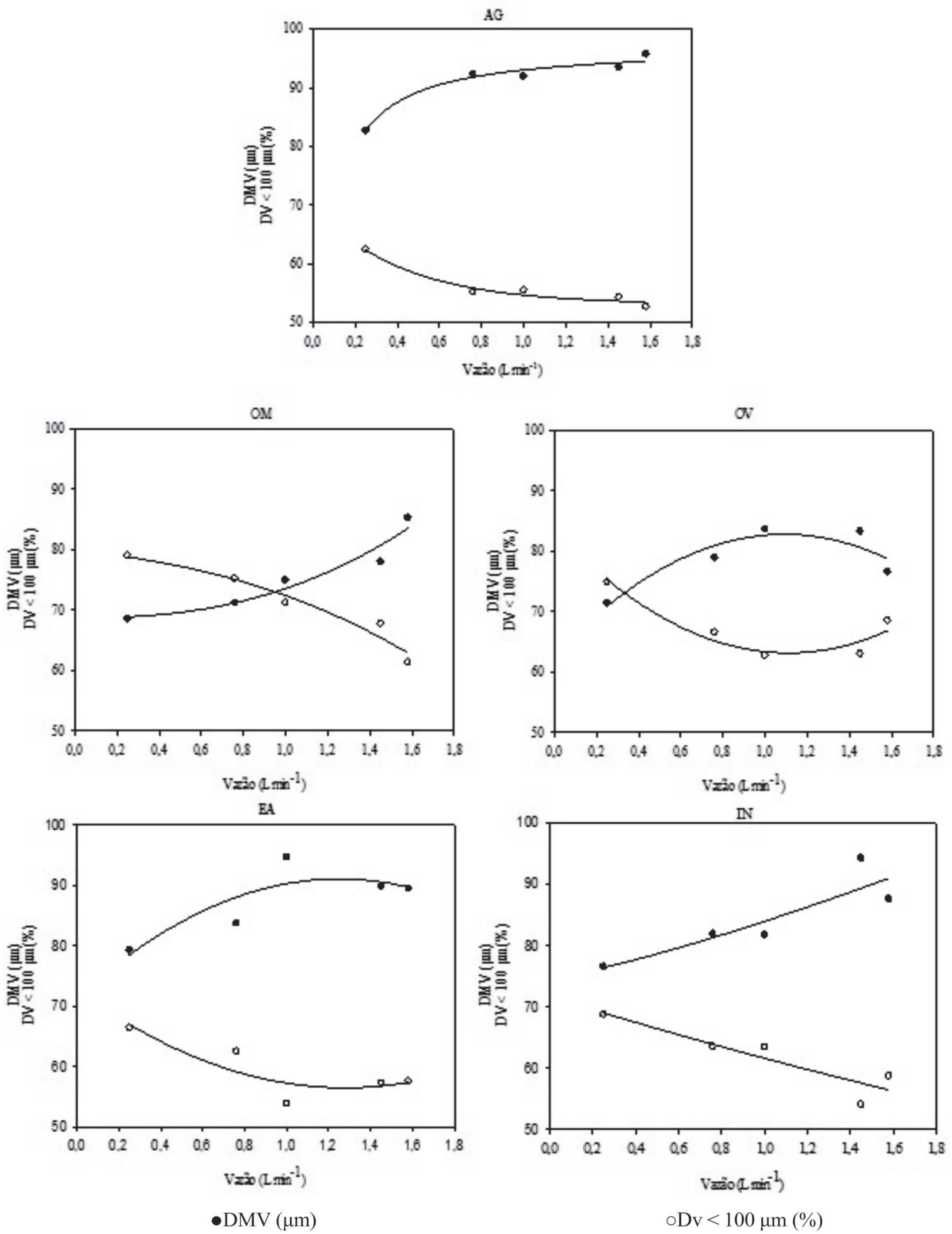

Figura 2: Diâmetro da mediana volumétrica (DMV) e percentagem do volume de gotas menores do que 100 ìm (Dv < 100 ìm) em função da vazão para as caldas a base de água (AG), óleo mineral (OM), óleo vegetal (OV), espalhante adesivo (EA) e inseticida (IN). 
Tabela 4: Equações, coeficientes de determinação $\left(\mathrm{R}^{2}\right)$, pontos críticos e valor de $\mathrm{F}$ calculado dos modelos de regressões representados na Figura 2

\begin{tabular}{|c|c|c|c|}
\hline Caldas & Equações & $\mathbf{R}^{2}(\%)$ & Fc \\
\hline \multirow{2}{*}{ AG } & DMV: $\mathrm{w}=97,1128 \mathrm{x} /(0,0435+\mathrm{x})$ & 96,33 & $26,18^{*}$ \\
\hline & $\mathrm{Dv}<100 \mu \mathrm{m}: \mathrm{w}=53,1986+16,8253 \mathrm{e}^{-2,3995 \mathrm{x}}$ & 95,95 & $10,86^{*}$ \\
\hline \multirow{2}{*}{$\mathrm{OM}$} & DMV: $\hat{y}=68,7088+4,8861 x^{2,4133}$ & 92,45 & $21,39 *$ \\
\hline & $\mathrm{Dv}<100 \mu \mathrm{m}: \mathrm{w}=83,0496 /\left(1+\mathrm{e}^{[-(x-2,4272) /-0,7440]}\right)$ & 95,01 & $35,44^{*}$ \\
\hline \multirow{4}{*}{$\mathrm{OV}$} & DMV: $\hat{y}=62,7769+36,7065 x-16,8208 x^{2}$ & 83,15 & $11,60^{*}$ \\
\hline & $\mathrm{X}_{\text {máx }}=1,09 \quad \mathrm{Y}_{\text {máx }}=82,80$ & & \\
\hline & $\mathrm{Dv}<100 \mu \mathrm{m}: \mathrm{w}=83,4392-36,5514 \mathrm{x}+16,4871 \mathrm{x}^{2}$ & 90,18 & $17,94^{*}$ \\
\hline & $\mathrm{X}_{\text {mín }}=1,11 \quad \mathrm{Y}_{\text {mín }}=63,18$ & & \\
\hline \multirow{4}{*}{ EA } & DMV: $\hat{y}=71,3987+31,5071 x-12,6029 x^{2}$ & 72,69 & $14,11 *$ \\
\hline & $X_{\text {máx }}=1,25 \quad Y_{\text {máx }}=91,09$ & & \\
\hline & $\mathrm{Dv}<100 \mu \mathrm{m}: \mathrm{w}=72,6681-25,3451 \mathrm{x}+9,9132 \mathrm{x}^{2}$ & 75,55 & $28,81^{*}$ \\
\hline & $\mathrm{X}_{\text {mín }}=1,28 \quad \mathrm{Y}_{\text {mín }}=56,47$ & & \\
\hline \multirow{2}{*}{ IN } & DMV: $\hat{y}=77,1557+14,3826 /\left(1-\mathrm{e}^{[-(x-1,0344) / 0,1830]}\right)$ & 80,44 & $13,29 *$ \\
\hline & Dv $<100 \mu \mathrm{m}: \hat{y}=74,7336+9,1885 \mathrm{x}^{1,2434}$ & 77,74 & $28,46^{*}$ \\
\hline
\end{tabular}

*Regressões significativas a 0,05 .

ciação numérica foi pequena, o que pode ter contribuído para a não influência na relação Q/M. Outra propriedade que pode ter influenciado na relação Q/M é a densidade das caldas, sobre as quais se nota que a da calda EA foi semelhante à da calda $\mathrm{AG}$. As caldas $\mathrm{OM}, \mathrm{OV}$ e IN, que apresentaram menores densidades, resultaram em maiores relações Q/M (Tabela 1 ).

Além disso, é possível verificar-se que a redução dos diâmetros das gotas pulverizadas acompanha o aumento da relação $\mathrm{Q} / \mathrm{M}$, isto é, as caldas que apresentaram os menores DMVs também resultaram em maiores relações Q/M. A calda EA, que apresentou relação Q/ M semelhante à da calda AG (Tabela 1), foi a que também gerou DMV e Dv < 100 ìm mais semelhantes aos da água (Figura 2). As caldas OM e OV, por sua vez, apresentaram os menores DMVs e mais se diferenciaram de $\mathrm{AG}$ neste aspecto, por isso a relação $\mathrm{Q} / \mathrm{M}$ foi maior nessas caldas.

Infere-se então que, neste trabalho, as diferenças encontradas de eficiência da eletrificação das caldas foram, além de influenciadas pelas vazões, provavelmente devidas às alterações dos espectros de gotas, e ao aumento da condutividade elétrica. Isso também foi defendido por Chaim et al. (2002), já que o tamanho das gotas influencia diretamente na relação $\mathrm{Q} / \mathrm{M}$, pois quanto menor os diâmetros das gotas, mais facilmente ocorre seu carregamento.

No entanto, é necessária cautela, pois se, por um lado, gotas pequenas proporcionam maior cobertura do alvo e favorecem sua eletrificação, por outro, são altamente susceptíveis à evaporação e à deriva, com risco de prejudicar a eficiência de controle, como afirmado por Sasaki et al. (2015) e Magno Júnior et al. (2014).
Por outro lado, se a pulverização eletrostática for empregada com critérios técnicos, atentando-se às condições meteorológicas, ao momento correto de aplicação e às características do alvo e da cultura, além de favorecer o manejo fitossanitário pode também levar a uma redução na taxa de aplicação, com possível redução da contaminação ambiental e dos operadores.

\section{CONCLUSÕES}

A distância entre o bocal do pulverizador e o alvo, a vazão e a composição da calda de aplicação influenciam a eficiência da pulverização eletrostática. O aumento da vazão de aplicação e o da distância entre o bocal do pulverizador e o alvo reduzem a relação $\mathrm{Q} / \mathrm{M}$ gerada pela pulverização eletrostática. Cada produto adicionado à calda de aplicação influencia de maneira peculiar a pulverização eletrostática, independentemente das características físico-químicas. A composição das caldas de aplicação e a vazão influenciam no espectro de gotas da pulverização pneumática.

\section{REFERÊNCIAS}

Carlton JB, Bouse LF \& Kirk IW (1995) Electrostatic charging of aerial spray over cotton. Transactions of the ASAE, 38:1641-1645.

Chaim A (1998) Aperfeiçoamento de bico de pulverização eletrostática para geração de gotas com alto nível de carga. Pesquisa Agropecuária Brasileira, 33:463-470.

Chaim A, Pessoal MCPY \& Ferracini VL (2002) Eficiência de deposição de agrotóxicos obtida com bocal eletrostático para pulverizador costal motorizado. Pesquisa Agropecuária Brasileira, 37:497-501.

Chaim A (2006) Pulverização eletrostática: principais processos utilizados para eletrificação de gotas. Jaguariúna, Embrapa Meio Ambiente. 17p. (Documentos, 57). 
Cunha JPAR, Alves GS \& Reis EF (2010) Efeito da temperatura nas características físico-químicas de soluções aquosas com adjuvantes de uso agrícola. Planta Daninha, 28:665-672.

Dobbins T (1995) Electrostatic spray heads convert knapack mistblowers to electrostatic operation. International Pest Control, 37:155-158.

Ferreira DF (2008) Sisvar: um programa para análises e ensaios de estatística. Revista Symposium, 6:36-41.

Laryea GN \& No SY (2005) Effect of fan speed and electrostatic charge on deposition of orchard canopy sprays. Atomization and Sprays, 15:133-144.

Magno Júnior RG, Teixeira MM, Fernandes HC, Rodrigues DE \& Alvarenga CB (2014) Desenvolvimento de um dispositivo eletrostático para pulverizador pneumático costal. Engenharia na Agricultura, 22:09-16.
Maski D \& Durairaj D (2010) Effects of charging voltage, application speed, target height and orientation upon charged spray deposition on leaf abaxial and adaxial surfaces. Crop Protection, 29:134-141.

Patel MK, Ghanshyam C, Reddy Mamidi VR \& Kapur P (2012) Performance and characterization of different material electrodes in electrostatic pesticide spraying nozzle system. International Journal of Applied Science \& Technology Research Excellence, 2:158-163.

Sasaki RS, Teixeira MM, Santiago H, Madureira RP, Maciel CFS \& Fernandes HC (2015) Adjuvantes nas propriedades físicas da calda, espectro e eficiência de eletrificação das gotas utilizando a pulverização eletrostática. Ciência Rural, 45:274-279.

Sokal RR \& Rohlf FJ (1995) Biometry: the principles and practice of statistics in biological research. $3^{\mathrm{a}} \mathrm{ed}$. New York, W. H. Freeman. 937p. 